\title{
THE EFFECT OF TACHYCARDIA ON THE BLOOD FLOW IN DOGS
}

\section{The Effect of Rapid Irregular Rhythms as Seen in AUricular Fibrillation}

BY HAROLD J. STEWART, J. HAMILTON CRAWFORD, AND A. B. HASTINGS (From the Hospital of the Rockefeller Institute for Medical Research, New York, N. Y.)

(Received for publication July 16, 1926)

The frequent occurrence of tachycardia, both regular and irregular, in patients makes it desirable to have exact information as to the effect of these rhythms on the circulation. Up to the present few studies have been directed toward this end either in patients or in experimental animals, because of the difficulty in the first place of obtaining samples of mixed venous blood and in the second place of inducing these rhythms under conditions which were not too far removed from normal conditions.

Stewart (1) in a study of the oxygen saturation of the arterial and of the venous arm blood in patients during auricular fibrillation and after the return to the normal rhythm under quinidine sulphate, found that during the period of fibrillation the arterial oxygen saturation was practically unchanged, while that of the venous blood was decreased. It appeared therefore that the blood flow was slower during auricular fibrillation than during the normal rhythm. Goldschmidt and Light (2) have shown that the oxygen saturation of the venous arm blood varies with temperature and to some extent with position, but the consistency in the changes which Stewart observed when these factors were taken into account make it seem unlikely that these two factors were responsible for them. Meakins (3) found that neither regular nor irregular tachycardia affected the oxygen saturation of the arterial blood in dogs. There is a difficulty however in the interpretation of Meakins' experiments, for the dogs were under paraldehyde anesthesia, the chests were open and the dogs were kept alive by means of artificial respiration. In cases 
where the rhythm is regular Carter and Stewart (4) have likewise reported marked anoxemia in a patient during paroxysms of auricular tachycardia and Dieuaide (5) has reported a similar state in a patient during attacks of paroxysmal ventricular tachycardia. On the other hand in Barcroft, Bock, and Roughton's (6) patient the oxygen saturation of the arterial blood was normal during paroxysmal auricular tachycardia. These variations in the observations so far reported leave the subject unsettled. In the work which we are now reporting we have studied the effect of induced tachycardia under physiological conditions and have been able to make the observations on the mixed venous blood. In this paper we shall report experiments on the effect of auricular fibrillation, as a type of irregular tachycardia, on the blood flow in normal dogs, while in a second paper we shall report other experiments on the effect of regular tachycardia.

\section{METHODS}

Dogs of diverse weights were used in these experiments. The animals were prepared 24 hours before the experiments by the operation which is now described. The operations were carried out under sterile precautions. Fther was given by the intratracheal method. With the dog lying well on his left side the skin and muscles were incised to expose the fourth right rib. About $6 \mathrm{~cm}$. of this rib was removed and the heart exposed through this opening. In the later experiments ample exposure of the heart was obtained simply by making an incision through the interspace between the fourth and the fifth ribs. A small sand bag placed under the dog's chest helped to give a good exposure. The lobes of the right lung were packed aside with squares of black japanese silk moistened with warm physiological salt solution. An incision $3 \mathrm{~cm}$. in length was made over the right auricle parallel to the auriculo-ventricular groove and about $0.5 \mathrm{~cm}$. above it. A special wire electrode was sutured to the auricular wall about $0.5 \mathrm{~cm}$. above the auriculoventricular groove (fig. 1). A second electrode was sutured $1.0 \mathrm{~cm}$. above the first. The wire electrodes were made of No. 30 gauge brass wire about $20.0 \mathrm{~cm}$. long. At one end the wire was coiled into a loose spiral of 8 to 10 turns. The spiral portion was $3.0 \mathrm{~mm}$. in diameter and $1.5 \mathrm{~cm}$. in length. The spiral ends were sutured to the auricle. The placing of the two spirals with reference to each other is illustrated in fig. 1. By this arrangement a short circuit is less likely to occur during stimulation through these electrodes. Three or four sutures of fine black silk doubled were sufficient to fix each electrode to the muscle. Fine curved needles were used. The four sutures were first placed in the auricular muscle; then the spiral electrode was held in place and the sutures tied about the spirals. Hemorrhage did not occur in placing the sutures in the auricular muscle. The wires 
leading from the spirals were insulated with small fine bore rubber tubing down to their attachment to the muscle. The pericardium was closed, the insulated wire brought outside the chest wall, and the chest closed tightly in layers after distension of the lungs to expel all free air from the pleural cavity. The whole operation required about three quarters of an hour from the time the administration of ether was begun until it was discontinued. The depth of anesthesia was kept as light as possible. The dogs were given morphine $32.0 \mathrm{mgm}$. hypodermically before being removed from the operating table. The dogs recovered from the ether within one to two hours. The operations were performed in the morning or early afternoon and by evening the dogs were walking about the cage. Care was exercised throughout the operation to prevent injury to the tissues.

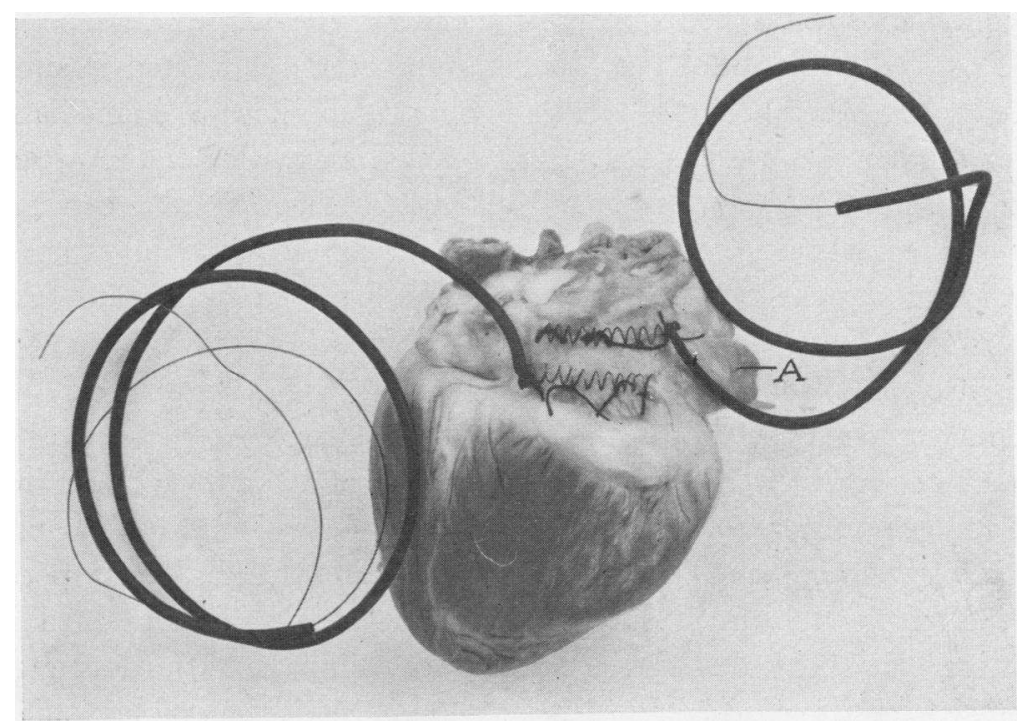

FIG. 1. This photograph shows the position in which the spiral wire electrodes are sutured to the right auricle. $A$ is the right auricular appendage.

\section{PLAN OF OBSERVATIONS}

The day after the preliminary operation the following experiment was performed. The dogs lay quietly on the animal table during these experiments without anesthesia. Non-polarizable electrodes were placed on the right fore leg and the left hind leg for the derivation of Lead II of the electrocardiogram. Samples of arterial and of mixed venous blood were drawn during the presence of the normal rhythm. The arterial blood was drawn from a femoral artery. The 
samples of mixed venous blood were obtained by inserting a special cannula into the right ventricle through the right external jugular vein (Stewart (7)). Novocaine 1 to 2 per cent was injected into the skin of the neck before exposing the vein. The right auricle was then stimulated through the wire electrodes by faradic current, and made to fibrillate for 30 to 90 minutes. The faradic current was obtained from one or two dry cell batteries in the primary circuit of a Du Bois-Reymond induction coil. At the end of the fibrillatory period and while the auricles were still fibrillating second samples of arterial and of mixed venous blood were drawn. Third samples were taken several hours after the end of the stimulation period. The oxygen contents of the blood samples were estimated in duplicate by the Van Slyke and Neill manometric method (8) immediately after the samples were taken. The oxygen capacities of the arterial and in some instances of the mixed venous blood were ascertained for the calculation of the degree of oxygen saturation. Electrocardiograms were made at the time the blood samples were taken in order to be certain of the cardiac rhythm then prevailing and to estimate the heart rate.

The oxygen consumed by the tissues per liter of blood was obtained by subtraction of the oxygen content of the mixed venous blood from the oxygen content of the arterial blood. By calculating the ratio of the oxygen consumed per liter of blood during the fibrillatory period to the oxygen consumed during the normal rhythm we obtained the relative blood flow during the induced rhythm, the ratio during the normal control period being placed at 100 . Since the dog lay quietly on the board during the experiment and had not been given food on the day of the experiment we have assumed that the changes in oxygen consumed per liter of blood were due to changes in blood flow, and not to changes in metabolism. We did not attempt to estimate the oxygen absorption of the dogs because of the wide variation in the results obtained in untrained, unanesthetized dogs. We therefore were unable to calculate in absolute figures the minute volume output by the heart or the output per beat.

\section{OBSERVATIONS}

We have studied the effect of auricular fibrillation on the blood flow in 10 dogs. Two additional experiments serve as controls. 
The effect of auricular fibrillation on the oxygen saturation of the arterial blood. The oxygen saturation of the arterial blood was unchanged in 4 observations, increased 4 per cent in one observation and decreased 2 to 3 per cent in 5 observations (table 1). None of these changes were of greater magnitude than the changes found in the two controls, namely, a 6 per cent increase and a 1 per cent decrease. On the whole therefore auricular fibrillation has no effect on the oxygen saturation of the arterial blood.

The effect of auricular fibrillation on the oxygen saturation of the mixed venous llood. The oxygen saturation of the mixed venous blood was decreased in every observation (table 1). The decrease varied between 12 and 35 per cent in 8 observations and was 7 and 9 per cent in the other two observations. With the return to the normal rhythm the saturation again increased. In dog 167 auricular fibrillation persisted, and in this instance the oxygen saturation of the mixed venous blood remained low. In the two controls there was an increase of 5 per cent in one and a decrease of 3 per centin the other.

The effect of auricular fibrillation on the llood flow. The blood flow in dog 157 was decreased 36 per cent during the period of auricular fibrillation, and began to return toward normal as soon as fibrillation stopped (table 1 and fig. 2). The rhythms which obtained at the time that the blood samples were taken in this dog were recorded electrocardiographically (fig. 3). In this case the return to normal rhythm did not take place at once, but passed through that of auricular flutter, a mechanism closely allied to fibrillation (fig. 3c). In $\operatorname{dog} 167$ (table 1), spontaneous auricular fibrillation was still present two and one half hours after discontinuance of the faradic stimulation; in this case the blood flow showed a further decrease. Similar decreases in blood flow occurred during auricular fibrillation in all of the 10 observations (table 1). The blood flow during the fibrillatory period was only 80 to 38 per cent of what it was during the normal rhythm, that is to say, the blood flow was decreased between 20 and 62 per cent. In 7 observations (dogs 157, 167, 169, 194, 197,198 , and 151) the blood flow was decreased more than 30 per cent; in the other 3 animals (dogs 193, 195, 199) the decrease was between 20 and 30 per cent. 


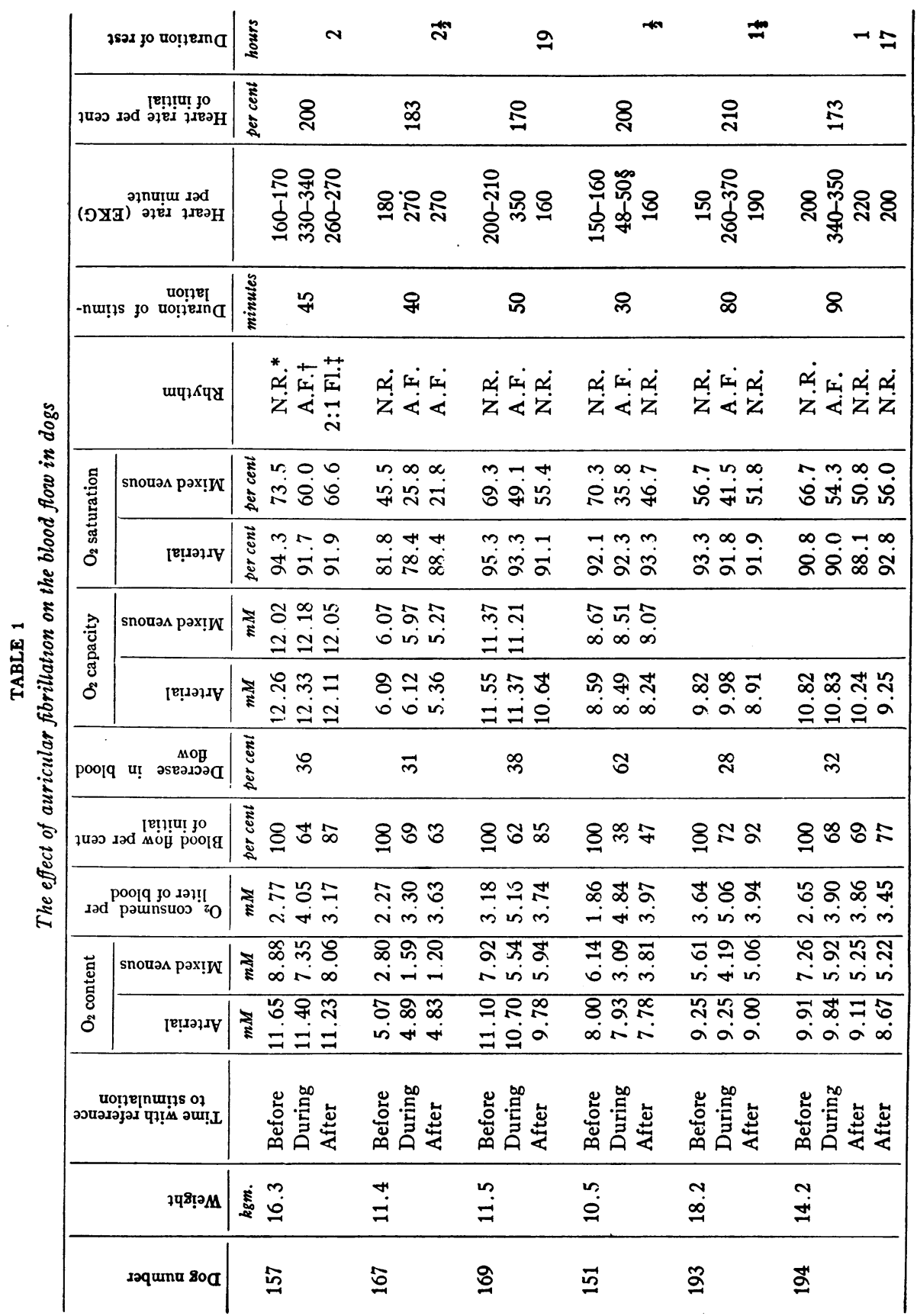




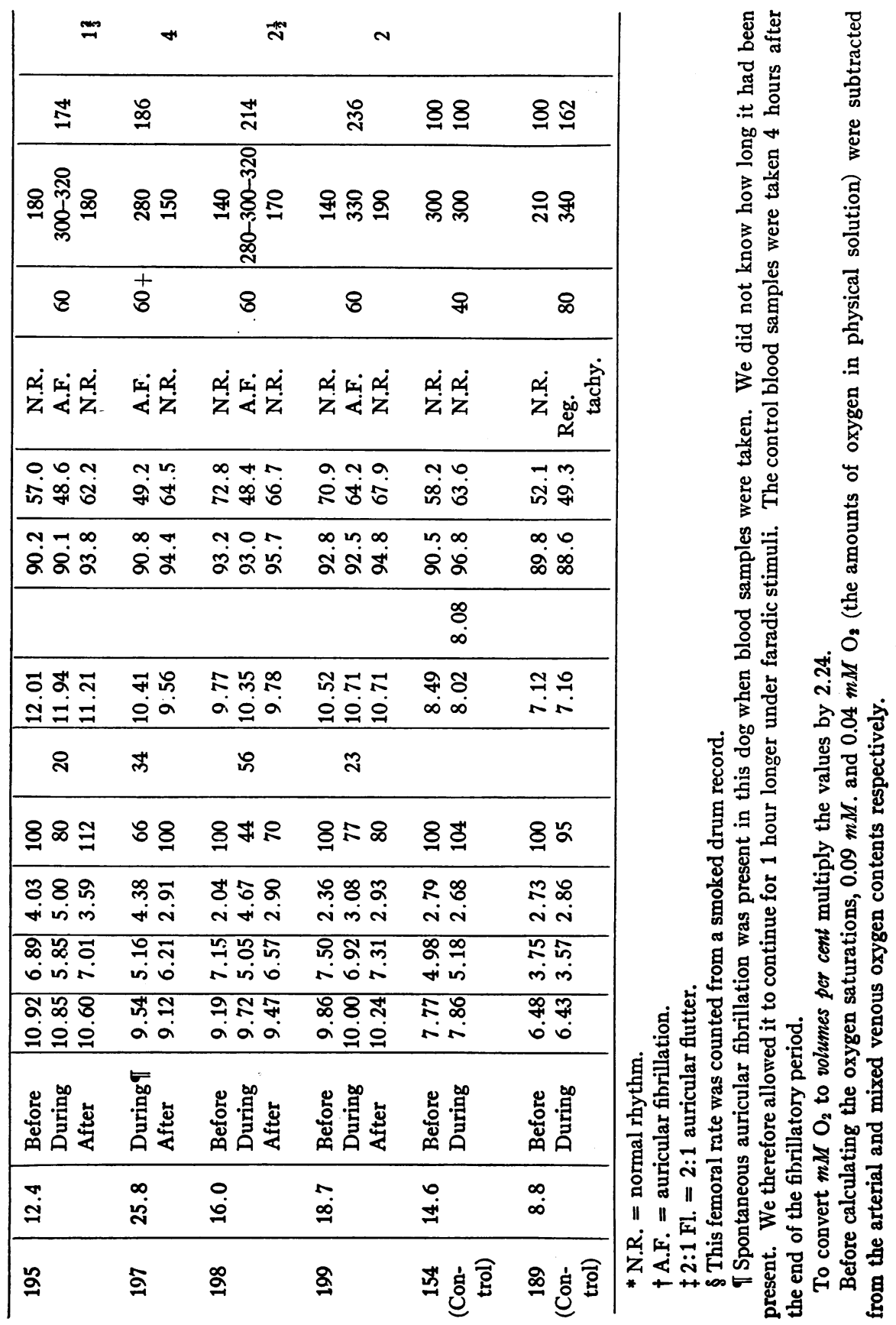




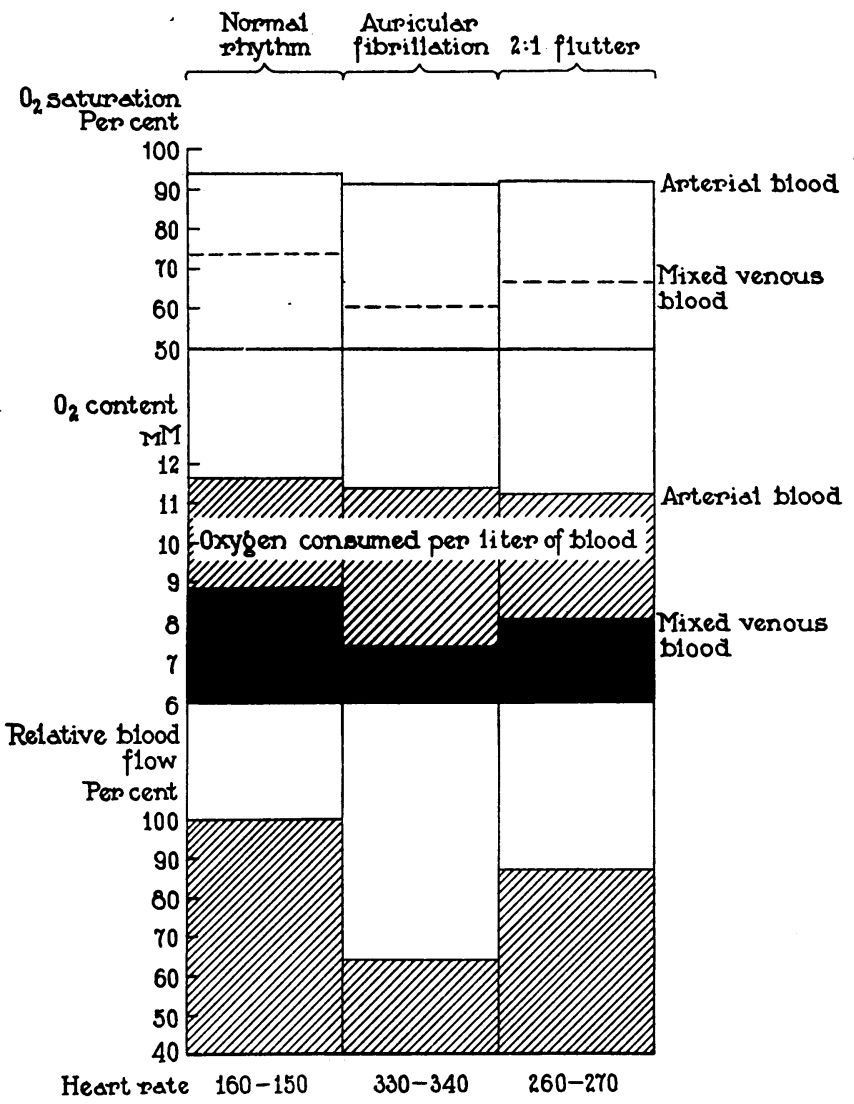

Fic. 2. The effect of irregular tachycardia (auricular fibrillation) on oxygen saturation of the arterial and of the mixed venous blood and on the blood flow in $\operatorname{dog} 157$ is indicated graphically in this figure. The oxygen saturation of the arterial blood was unchanged during the fibrillatory period, while that of the mixed venous blood was decreased. The oxygen consumed by the tissues per liter of blood was increased; the blood flow was decreased 36 per cent. When fibrillation stopped the blood flow increased, the oxygen consumed per liter of blood decreased, and the oxygen saturation of the mixed venous blood increased. The return to normal rhythm did not take place at once, but passed through that of auricular flutter (fig. 3). The ventricular rate prevailing at the time the blood samples were taken is shown at the bottom of the figure. 
Dog 154 (table 1) serves as a control. After stimulation for 1 hour the blood flow had not changed and on analysis of the electrocardiographic records it was found that the rhythm had not been influenced

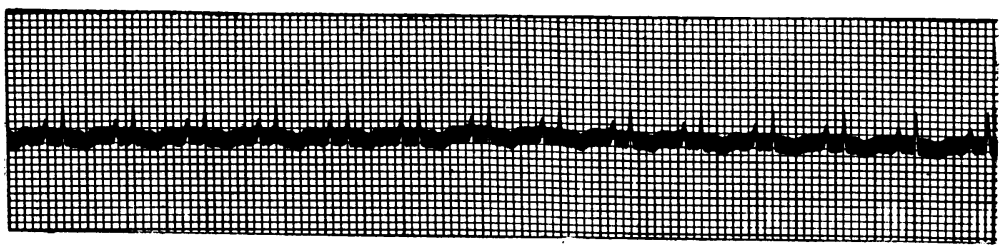

a

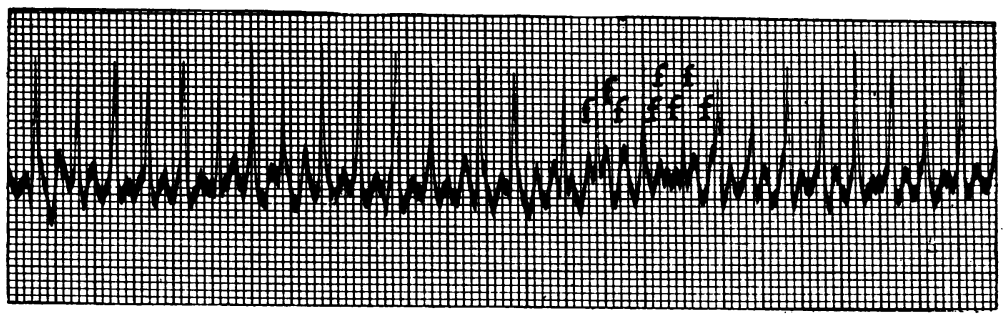

b

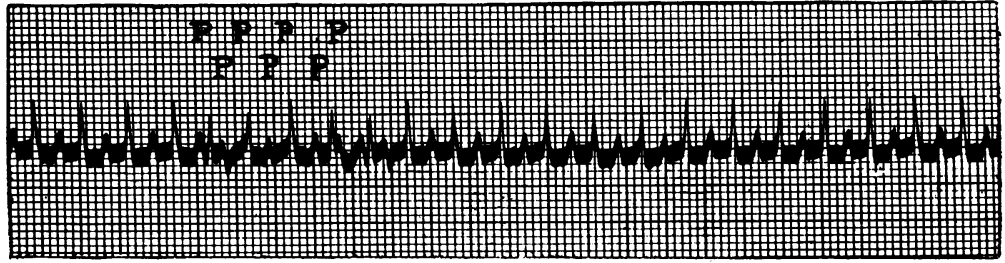

C

FIG. 3. Electrocardiograms of dog 157 are shown at the time the blood samples were drawn $3 \mathrm{a}$ was made during the normal rhythm, $3 \mathrm{~b}$ during auricular fibrillation ( $f f f$ are fibrillation waves). $3 c$ was taken 2 hours after the end of stimulation and shows that $2: 1$ auricular flutter was present ( $P P P$ are flutter waves). Lead II of the electrocardiogram is reproduced. Divisions of the ordinates equal $10^{-4}$ volts. Divisions of the abscissae equal 0.04 of a second. The original curves are sharply contrasted black and white; no half tones are lost by the method of reproduction here used. The electrocardiograms are reduced to two-thirds of their natural size.

by the stimulation. At autopsy it was found that one of the wire electrodes was broken inside the rubber insulating tube so that the circuit was broken and current did not reach the heart. Dog 189 (table 1) is the other control. During faradic stimulation a regular 
tachycardia developed instead of auricular fibrillation. In this dog there was no change in blood flow.

In these experiments then the blood flow was decreased in 10 dogs during auricular fibrillation while the two controls failed to show such a change.

\section{DISCUSSION}

The results of these experiments indicate that the blood flow is decreased during auricular fibrillation. This conclusion was arrived at from a comparison of the oxygen consumed by the tissues per liter of blood during the normal rhythm and during auricular fibrillation. The dogs lay quietly on the board during the experiment without anesthesia or sedatives. They were given no food on the day of the experiment. Under these conditions the tissue requirements in all probability remain unchanged. On the basis of these considerations we believe that we are justified in interpreting the changes in the oxygen consumed as being due to changes in blood flow. We think of the events as follows. If the oxygen requirements of the tissues remain unchanged and the same amount of blood is brought to a given part in a unit of time, the same amount of oxygen should be removed from the blood, while if the blood flow is slower more oxygen should be removed. It would have been desirable to ascertain the minute volume output of the heart or the output of the heart per beat rather than the change in blood flow, but for making this calculation we have not the requisite data. To obtain this information it would have been necessary to measure the oxygen absorbed per minute by the dogs during each variety of cardiac rhythm. But to do so masks must be put on the dogs. When they are untrained and unanesthetized there is in consequence a disturbance which brings about wide variation in the results. On the other hand we did not wish to anesthetize the animals because of the secondary changes that accompany anesthesia. Because of the undesirable effect of morphine on the respiration we decided against the use of this drug.

The results of these experiments are in harmony with the results which Blumgart and Weiss (9) have obtained in patients by another method. They measured the time required for radium " $\mathrm{c}$ " to pass from the vein of one arm to the artery of the other arm (circulation time) and found that this is much increased in auricular fibrillation. 
We are next concerned with analyzing the reason for the decreased blood flow during auricular fibrillation. Among the factors that may be operative is the ineffectiveness of many of the ventricular beats which fail of expelling blood into the arteries, that is to say a pulse deficit may be present. We know that a pulse deficit is present during auricular fibrillation in many patients; it seems likely that it may also occur during experimental conditions. Experiments are now in progress to ascertain this point.

In spite of the fact that during auricular fibrillation the mixed venous blood reaching the lungs is much more unsaturated than during the normal rhythm, the oxygen saturation of the arterial blood is unchanged. This state of affairs is due probably to the combination of two factors. In the first place under normal conditions the mixed venous blood is quickly saturated with oxygen in the lungs with time to spare to take care of blood that is even more unsaturated. In the second place, since in auricular fibrillation the blood flow is decreased 20 to 62 per cent, the blood remains in the lungs a correspondingly longer time and in this way the more unsaturated mixed venous blood is raised to the same level of saturation as obtains during the normal rhythm. Stewart (10) has found that there is no change in the oxygen saturation of the arterial blood in patients with auricular fibrillation when the ventricular rate is increased following the injection of atropine.

In chronic cardiac disease the hemoglobin content of the blood is often increased. A compensatory mechanism is in this manner brought about which facilitates the transport of oxygen. In patients a long time is required for its development. In these experiments there was no consistent change to correspond with this in the hemoglobin content (oxygen capacity) of the blood during auricular fibrillation.

\section{SUMMARY}

The blood flow and oxygen saturations of the arterial and of the mixed venous blood has been studied during experimental auricular fibrillation in 10 normal unanesthetized dogs. It was found that:

1. The blood flow decreased 20 to 62 per cent during auricular fibrillation. 
2. The oxygen saturation of the arterial blood was unchanged during auricular fibrillation.

3. The oxygen saturation of the mixed venous blood decreased during auricular fibrillation.

4. Following the return of the heart to the normal rhythm the oxygen saturation of the mixed venous blood and the blood flow return toward normal. In one instance in which spontaneous auricular fibrillation persisted there was no such tendency.

5. The changes described as occurring during auricular fibrillation did not occur in two control experiments.

\section{CONCLUSIONS}

1. The heart during auricular fibrillation is less effective in the propulsion of blood than it is during the normal slow rhythm.

2. Auricular fibrillation per se does not affect the oxygen saturation of the arterial blood.

\section{BIBLIOGRAPHY}

1. Stewart, H. J.: Arch. Int. Med., 1923, xxxi, 871. Observations on the Blood Gases in Auricular Fibrillation and after the Restoration of the Normal Mechanism.

2. Goldschmidt, S., and Light, A. B.: Amer. Jour. Physiol., 1925, lxxiii, 146. The Effect of Local Temperature upon the Peripheral Circulation and Metabolism of Tissues as Revealed by the Gaseous Content of the Venous Blood.

Goldschmidt, S., and Light, A. B.: Amer Jour. Physiol., 1925, lxxiii, 127. A Comparison of the Gaseous Content of Blood from Veins of the Forearm and the Dorsal Surface of the Hand as Indicative of Blood Flow and Metabolic Differences in These Parts.

3. Meakins, J.: Heart, 1921-22, ix, 185. The Influence of Circulatory Disturbances on the Gaseous Exchange of the Blood. I. The Oxygen Saturation of the Arterial Blood in Tachycardia.

4. Carter, E. P., and Stewart, H. J.: Arch Int. Med., 1923, xxxi, 390. Studies of the Blood Gases in a Case of Paroxysmal Tachycardia.

5. Dieuaide, F. R.: Johns Hop. Hosp. Bull., 1924, xxxv, 229. Observations on the Respiratory Gases in Ventricular Paroxysmal Tachycardia.

6. Barcroft, J., Bock, A. V., and Roughton, F. J.: Heart, 1921-22, ix, 7. Observations on the Circulation and Respiration in a Case of Paroxysmal Tachycardia. 
7. Stewart, H. J.: Jour. Biol. Chem., 1925, lxii, 641. The Oxygen and Carbon Dioxide Contents of the Arterial and Mixed Venous Blood in Normal Intact Dogs.

8. Van Slyke. D. D., and Neill, J M.: Jour. Biol. Chem., 1924, lxi, 523. The Determination of Gases in Blood and Other Solutions by Vacuum Extraction and Manometric Measurement. I.

9. Blumgart, H., and Weiss, S.: Jour. Clin. Invest., 1926, ii, 600. The Velocity of Blood Flow in Health and Disease.

10. Stewart, H. J.: Jour. Clin. Invest., 1926, iii, 241. The Effect of Increased Heart Rate Due to the Injection of Atropine on the Oxygen Saturation of the Arterial and Venous Blood of Patients with Heart Disease. 